\title{
Embryos of non-native anoles are robust to urban thermal environments
}

\author{
Sarin Tiatragul ${ }^{\mathrm{a}, \mathrm{b}, *}$, Audeline Kurniawan ${ }^{\mathrm{c}}$, Jason J. Kolbe ${ }^{\mathrm{d}}$, Daniel A. Warner ${ }^{\mathrm{a}, \mathrm{c}}$ \\ a Department of Biological Sciences, Auburn University, Auburn, AL 36849, United States \\ b Department of Biology, Gonzaga University, Spokane, WA 99258, United States \\ c Department of Biology, University of Alabama at Birmingham, Birmingham, AL 35294, United States \\ d Department of Biological Sciences, University of Rhode Island, Kingston, RI 02881, United States
}

\section{A R T I C L E I N F O}

\section{Keywords:}

Anolis

Developmental plasticity

Invasive species

Lizard

Urban adaptation

\begin{abstract}
A B S T R A C T
The transformation of natural habitats into urban landscapes dramatically alters thermal environments, which in turn, can impact local biota. Ectothermic organisms that are oviparous are particularly sensitive to these altered environments because their embryos cannot behaviorally thermoregulate and the surrounding environment determines the temperature experienced during development. We studied the effects of urban and forested thermal environments on embryo development and hatchling phenotypes in two non-native lizards (Anolis sagrei and A. cristatellus) in metropolitan Miami, Florida. To determine if embryos from urban and forested sites are adapted to their respective thermal environments, we incubated eggs from each site using temperatures that simulate likely nest conditions in both urban and forested environments. For both species, urban thermal environments accelerated embryonic development, but had no impact on egg survival or any of the phenotypic traits that were measured (e.g., body size, running performance, and locomotor behavior). Our results provide no evidence that embryos from urban and forested sites are adapted to their respective thermal environments. Instead, the lack of any major effects suggest that embryos of both species are physiologically robust with respect to novel environments, which could have facilitated their success in establishing in nonnative ranges and in human-modified landscapes.
\end{abstract}

\section{Introduction}

Urbanization results in numerous physical changes to the environment that can dramatically impact organisms, populations, and ecosystems (Shochat et al., 2006; Forman, 2014). Temperature is an important abiotic factor that is consistently altered when natural habitat is converted to an urban landscape. Indeed, the urban heat island effect is characterized by markedly higher temperatures (as high as $12{ }^{\circ} \mathrm{C}$ increase) in cities compared to nearby natural areas due to the reduction in tree canopy and solar radiation being retained in materials like asphalt and concrete (Rizwan et al., 2008; Brazel et al., 2000; Tereshchenko and Filonov, 2001). Thermal changes due to urbanization can have substantial effects on the local biota (e.g., Frankie and Ehler, 1978; Hamer and Parris, 2011), and in many cases organisms have responded positively to urban temperatures. For example, native ant species in North Carolina have increased in abundance in warm/ open disturbed areas in cities (Menke et al., 2011), and larval survival of some European butterflies is increased under urban temperatures (Kaiser et al., 2016). Indeed, dramatic changes in temperature in urban environments have the potential to exert a substantial ecological and evolutionary pressure on organisms that inhabit cities.

Adaptation or acclimation to novel thermal environments (such as those in urban areas) can occur across all life stages, from embryos to adults (Angilletta et al., 2004). However, most studies of thermal adaptation have focused primarily on adult stages, rather than early developmental periods (Angilletta et al., 2002; Angilletta, 2009; but see Oufiero and Angilleta, 2006; Du et al., 2010). Early developmental stages are important because (1) embryos cannot behaviorally thermoregulate to the same extent as adults, and (2) phenotypic development is very sensitive to environmental conditions. Oviparous ectotherms that lack parental care may be particularly sensitive to developmental environments because embryos have a limited capacity to adjust their immediate temperature (Telemeco et al., 2016), and surrounding environments largely determine egg incubation temperatures after oviposition. Thus, exposure to novel embryonic environments may be a cause of rapid physiological adaptation. For example, many reptiles and fish from cool climates have embryos that develop faster than those from warm climates when incubated under the same controlled temperature (Conover and Schultz, 1995; Angilletta et al., 2002; Ewert et al., 2005; Niewiarowski and Angilletta, 2008; Du et al.,

\footnotetext{
* Corresponding author at: Department of Biological Sciences, Auburn University, Auburn, AL 36849, United States.

E-mail addresses: stiatragul@auburn.edu (S. Tiatragul), audeline@uab.edu (A. Kurniawan), jjkolbe@uri.edu (J.J. Kolbe), dan.warner@auburn.edu (D.A. Warner).
} 
2010), providing evidence for local adaptation to thermal environments. These examples illustrate countergradient variation, which occurs when genetic influences act against environmental effects such that populations of the same species exposed to different conditions react to their environmental stressors differently. Importantly, adaptation to thermal environments during development may be further influenced by maternal nesting behavior (Angilletta et al., 2004; Doody et al., 2006). Thus, studies that assess the effects of urban versus natural environments on embryos from those respective locations will be highly informative for understanding how these organisms might cope in human-modified landscapes.

Lizards of the genus Anolis have been useful models for studies of adaptation and acclimation to novel environments (e.g., Huey et al., 2003; Kolbe et al., 2012, 2014; Stuart et al., 2014), including urban areas (Kolbe et al., 2015; Winchell et al., 2016). Many Anolis species have experienced rapid environmental change during biological invasions and habitat modification such as urbanization. However, nearly all studies of adaptation to local environments in Anolis have focused on adult stages (Losos et al., 2004; Kolbe et al., 2012; but see Kolbe et al., 2014), and rarely assess plastic responses of embryos to novel environments in controlled experiments. The lack of research on developmental plasticity of Anolis embryos may be partially due to little information about natural nest conditions. Indeed, the extent of current knowledge of Anolis nesting behavior is that females select a variety of microhabitats for nests (Rand, 1967; Andrews, 1988) and prefer relatively moist environments (Socci et al., 2005; Reedy et al., 2013). To our knowledge, nothing is published about the thermal conditions of natural nests in the field.

We studied the effects of incubation temperatures on development and hatchling phenotypes of two non-native Anolis lizard species: the Cuban brown anole (Anolis sagrei) and the Puerto Rican crested anole (Anolis cristatellus). Both species are found in urban and forested sites across their non-native ranges in Miami-Dade County, Florida. These Anolis species occur in high densities in urban and forested areas in Miami (Kolbe et al., 2016). We sampled populations of both species from urban and forested areas, and housed individuals under a common laboratory environment. The eggs produced by females from both sites were assigned to incubation temperature regimes that mimicked potential nest sites in both sites to determine how embryos from urban and forest populations respond to the developmental temperatures in these respective environments. If anole embryos from urban or forested sites are adapted to their respective environments, then we predict that urban incubation temperature would be beneficial to embryos from urban environments, but detrimental to those from forested environments, and vice versa for the effects of incubation temperature in forested areas (i.e., population $x$ temperature interaction). However, if anole embryos from each site respond to urban and forested thermal environments in similar ways, then this finding would rule out local adaption. Lastly, if incubation temperature does not contribute to variation in phenotypic development, then this finding would also suggest that Anolis embryos are not adapted to urban temperatures, but are physiologically robust to novel environments, which might facilitate their ability to establish in non-native ranges or in human-modified landscapes.

\section{Material and methods}

\subsection{Lizard collection and husbandry}

Both Anolis species were collected from urban and forested areas in Miami-Dade County and shipped to the University of Alabama at Birmingham. The forested areas consist of large fragments of dense forest within an urban matrix, and are structurally and thermally different from the urban collection site (Kolbe et al., 2016). On 17 March 2015, adult $A$. cristatellus were collected at an urban site along Red Road in South Miami, FL (14 females, 7 males) and at a forested site at Matheson Hammock Park (10 females, 5 males). Additional lizards from these urban ( 5 females, 3 males) and forested ( 9 females, 4 males) sites were collected on 21 May 2015. On 1 July 2015, adult $A$. sagrei were collected from the same urban site (11 females, 6 males), and a nearby forested site at Montgomery Botanical Center (11 females, 6 males). The urban site was located $1.2 \mathrm{~km}$ from the forested site at Matheson Hammock Park and $1.6 \mathrm{~km}$ from the site at Montgomery Botanical Center. These two species occur sympatrically at the urban site, but occur alone at the forested sites, $A$. cristatellus at Matheson Hammock Park and A. sagrei at Montgomery Botanical Center.

These lizards formed a captive breeding colony. Females were housed in cages $(29 \mathrm{~cm}$ high $\times 26 \mathrm{~cm}$ wide $\times 39 \mathrm{~cm}$ deep) containing a small pot filled with moist soil to encourage oviposition, and two perches and an artificial plant used for perching and hiding. Males were rotated among cages weekly to ensure egg fertility and to minimize any potential bias due to paternity; each female was exposed to most of the males used in this study, but males and females from different populations were never housed together. Cages were illuminated with ReptiSun 5.0 UVB bulbs (ZooMed Inc.) on a 12-h light: dark cycle. Daily room temperature was set at $26.7^{\circ} \mathrm{C}$. Due to space limitations, at a given time there were 12 cages of the $A$. sagrei breeding colony that were not exposed to ReptiSun 5.0 UVB bulbs, but cages were rotated weekly to eliminate cage location effects. Lizards were fed live crickets (dusted in a mix of calcium and vitamins) three times per week and cages were misted with water daily to maintain high humidity.

\subsection{Experimental design}

From 1 May to 21 August 2015, pots from each cage were thoroughly checked three times per week for freshly laid eggs. After collection, eggs were immediately weighed (to $0.0001 \mathrm{~g}$ ) and placed individually in glass jars filled with moist vermiculite $(-150 \mathrm{kPa})$ and covered with plastic wrap (sealed with a rubber band) to prevent evaporation. Eggs were allocated to one of two incubation treatments that mimicked thermal regimes of potential nest sites in urban and forested sites. Because Anolis lizards produce approximately one egg every 7-10 days, eggs were allocated to each treatment such that successive eggs produced by a given female were alternated between urban and forested incubation temperatures. This allocation of eggs to treatments minimized any potential biases associated with the order of egg production.

To determine the temperature regimes for the incubation treatments, thermal data were collected at each site. Temperature loggers (iButtons) were placed in potential nest sites in urban (Red Road, $n=3$ ) and forested areas (Matheson, $\mathrm{n}=5$ ). The canopy openness over nest sites at the urban location ranged from $20 \%$ to $90 \%$ (mean=42\%), and from $1 \%$ to $10 \%($ mean $=5 \%)$ at the forested location. This difference reflected the relatively open canopy at the urban site compared to the very dense canopy at the forested sites, rather than a biased-selection of potential nest sites. Thus, the potential nest sites chosen were likely within the range of conditions that lizards choose among at each location. All iButtons were placed at $4-6 \mathrm{~cm}$ depth in the soil. From thermal data collected from 1 June - 15 Sept 2014 at each potential nest site, we calculated the average temperature for each hour of the day. The daily thermal profile (based on hourly averages) for urban and forested sites (Fig. 1) was uploaded into programmable incubators (Memmert IPP 400 incubators) and looped daily for the duration of the study. Thus, the incubation temperatures mimicked an average daily thermal regime that eggs would likely experience in urban and forested areas in the field. This $2 \times 2$ experimental design (two temperature fluctuation regimes and two different populations) enabled us to evaluate if embryos from different sites differ in their response to urban vs forested incubation temperature regimes.

Eggs were checked daily for hatchlings. For each hatchling, we recorded the date of hatching, body mass (to $0.0001 \mathrm{~g}$ ), snout-vent 


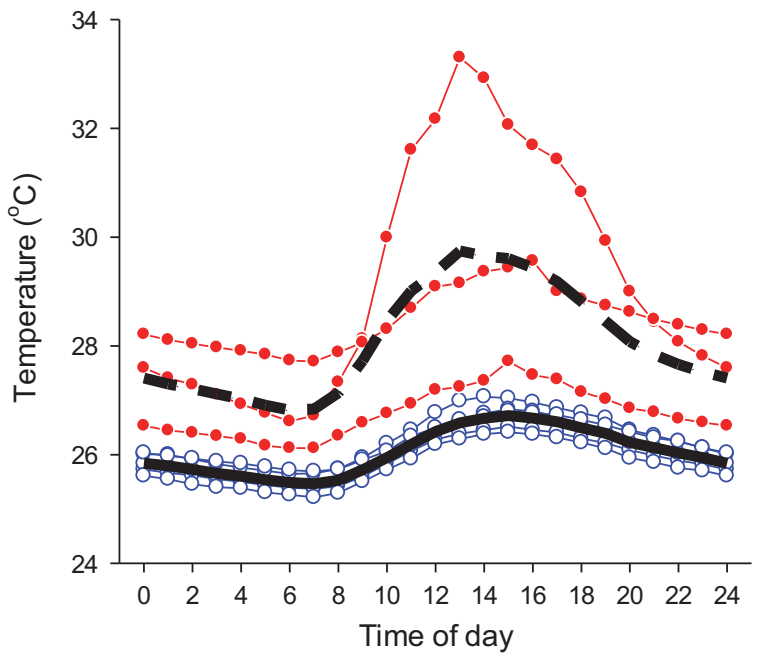

Fig. 1. Daily temperature fluctuations at potential nest sites in urban and forested areas in Miami-Dade County. The open/blue circles represent average hourly thermal profiles for each nest in the forested site, and the closed/red circles represent average hourly thermal profiles for each nest in the urban site. The hourly thermal regimes (repeated every day during incubation) for the egg incubation study are represented by the thick dashed line (urban site) and thick solid line (forested site).(For interpretation of the references to color in this figure legend, the reader is referred to the web version of this article.)

length (SVL) to $1 \mathrm{~mm}$, and tail length (TL) to $1 \mathrm{~mm}$. The sex of $A$. sagrei was identified based on dorsal pattern and the presence (male) or absence (female) of enlarged scales posterior to the cloaca; the sex of hatchling $A$. cristatellus was not identified because sexually dimorphic characters are not present in this species. All hatchlings were marked with unique toe-clips before being individually housed in small cages. Hatchling cages $(13 \mathrm{~cm}$ high $\times 21 \mathrm{~cm}$ wide $\times 17 \mathrm{~cm}$ deep) contained one perch and some artificial leaves for perching. Hatchlings were fed three times a week with small crickets and misted with water daily. At about 1 week after hatching, we measured locomotor performance of each lizard on a $1-\mathrm{m}$ long electronically timed race track set at a $20^{\circ}$ angle. The race track contained infra-red sensors (spaced at $25-\mathrm{cm}$ intervals) connected to a computer, which enabled us to quantify running speed over relatively short $(25 \mathrm{~cm})$ and long $(1 \mathrm{~m})$ distances. Each individual was encouraged to run along the racetrack with a soft paint brush and each lizard was run five times. Only the fastest $25-\mathrm{cm}$ and $1-\mathrm{m}$ speeds (over all the trials for an individual) were used in the statistical analyses. During running trials, the number of pauses that lizards made over the $1-\mathrm{m}$ track were counted. Because some individuals showed no motivation to run during any trial, locomotor behavior of individuals was classified as either "ran" versus "did not run." All running trials were conducted at $28^{\circ} \mathrm{C}$.

\subsection{Statistical analysis}

All data were analyzed with SAS software. All dependent variables were inspected for normality and log-transformed to improve normality when necessary. Data sets for each species were analyzed separately. The sexes were combined for analyses of the A. sagrei data set because preliminary results demonstrated no differences between males and females for any trait measured (all p-values >0.144). A generalized linear model with Poisson distribution was used to evaluate the effect of site on egg production. Generalized linear mixed models with a binomial error structure were used to quantify the effects of site of origin (i.e., urban vs forested), incubation thermal regime (urban vs forested), and their interaction on egg survival, hatchling survival, and locomotor behavior (ran vs did not run). Mixed model analyses of variance or covariance were used to quantify these same effects on incubation length, SVL, TL, body mass, locomotor performance, and the number of stops when running over $1 \mathrm{~m}$. The number of stops made by lizards was calculated as the average across the five running trials. The covariates used in each analysis are listed in Table 1. Maternal identity was used as a random effect in every model.

\section{Results}

The number of eggs produced by females did not differ between sites (A. sagrei, $\mathrm{F}_{1,20}=1.5, \mathrm{p}=0.228 ; A$. cristatellus, $\mathrm{F}_{1,32}=0.9$, $\mathrm{p}=0.348)$. Egg survival was relatively high for A. sagrei (89\%), but substantially lower for A. cristatellus (51\%) regardless of incubation treatment or site of origin (Tables 1 and 2). Incubation temperature influenced incubation period in the same direction for eggs from both sites (i.e., urban vs forested; Tables 1 and 2, Fig. 2). Eggs incubated under a thermal regime that mimicked an urban environment hatched sooner than those incubated under a forested thermal regime, and this pattern was similar for both $A$. sagrei and A. cristatellus.

Site of origin, incubation temperature, and their interaction had minimal, if any, effect on the morphological, performance, or behavioral traits for either species (Tables 1 and 2; Figs. S1 and S2). The only traits that were significantly influenced by incubation temperature was SVL and mass for A. cristatellus; offspring from the urban incubation treatment were only $0.4 \mathrm{~mm}$ shorter and $0.007 \mathrm{~g}$ lighter than those from the forested incubation treatment. Of the eggs that hatched, most hatchlings survived until they were tested for locomotor

Table 1 .

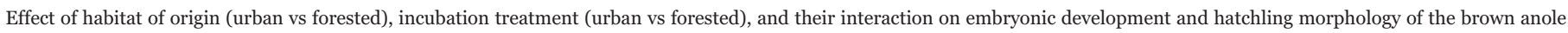

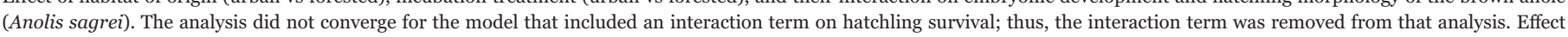
sizes $(\beta \pm \mathrm{SE})$ were calculated from each analysis, which included transformed data in most cases. Covariates: *egg mass, ${ }^{* *}$ snout-vent length, ${ }^{* *}$ body mass just prior to racing.

\begin{tabular}{|c|c|c|c|c|c|c|c|c|c|c|}
\hline \multirow[t]{2}{*}{ Dependent variable } & \multicolumn{4}{|c|}{ Habitat } & \multicolumn{3}{|l|}{ Treatment } & \multicolumn{3}{|l|}{ Interaction } \\
\hline & $\mathrm{df}$ & $\beta \pm \mathrm{SE}$ & $\mathrm{F}$ & $\mathrm{p}$ & $\beta \pm \mathrm{SE}$ & $\mathrm{F}$ & $\mathrm{p}$ & $\beta \pm \mathrm{SE}$ & $\mathrm{F}$ & $\mathrm{p}$ \\
\hline Incubation Period & 1,62 & $0.009 \pm 0.024$ & 1.8 & 0.185 & $0.166 \pm 0.016$ & 284.6 & $<0.001$ & $0.040 \pm 0.022$ & 3.3 & 0.073 \\
\hline Egg survival & 1,87 & $1.784 \pm 1.200$ & 1.1 & 0.306 & $-0.021 \pm 0.712$ & 2.6 & 0.129 & $-2.004 \pm 1.334$ & 2.3 & 0.137 \\
\hline Snout-vent length $(\mathrm{mm})^{*}$ & 1,69 & $0.015 \pm 0.014$ & 0.8 & 0.364 & $0.018 \pm 0.011$ & 2.6 & 0.111 & $-0.009 \pm 0.016$ & 0.4 & 0.549 \\
\hline Mass $(\mathrm{g})^{*}$ & 1,69 & $0.038 \pm 0.028$ & 1.7 & 0.197 & $0.022 \pm 0.023$ & 0.8 & 0.374 & $-0.015 \pm 0.033$ & 0.2 & 0.645 \\
\hline Condition, Mass (g)** & 1,69 & $0.027 \pm 0.029$ & 0.8 & 0.381 & $0.010 \pm 0.026$ & 0.0 & 0.834 & $-0.013 \pm 0.036$ & 0.1 & 0.728 \\
\hline Tail Length (mm) & 1,69 & $0.019 \pm 0.020$ & 0.2 & 0.667 & $0.007 \pm 0.015$ & 0.2 & 0.646 & $-0.024 \pm 0.021$ & 1.3 & 0.264 \\
\hline \multicolumn{11}{|l|}{ Running performance } \\
\hline Over $25 \mathrm{~cm}^{* * *}$ & 1,61 & $0.463 \pm 0.243$ & 3.3 & 0.075 & $0.044 \pm 0.207$ & 0.1 & 0.750 & $-0.180 \pm 0.289$ & 0.4 & 0.535 \\
\hline Over $1 \mathrm{~m}^{* * *}$ & 1,59 & $-0.007 \pm 0.183$ & 0.0 & 0.881 & $-0.075 \pm 0.183$ & 0.1 & 0.717 & $0.057 \pm 0.255$ & 0.1 & 0.824 \\
\hline Number of stops & 1,62 & $-1.400 \pm 1.531$ & 0.3 & 0.559 & $-1.370 \pm 1.616$ & 0.3 & 0.577 & $1.476 \pm 2.257$ & 0.4 & 0.516 \\
\hline locomotor behavior & 1,62 & $-0.268 \pm 0.621$ & 0.7 & 0.413 & $-0.613 \pm 0.685$ & 2.2 & 0.139 & $-0.307 \pm 1.024$ & 0.1 & 0.765 \\
\hline Growth & 1,12 & $-0.385 \pm 0.559$ & 1.1 & 0.310 & $-1.367 \pm 0.632$ & 1.8 & 0.208 & $1.642 \pm 0.821$ & 4.0 & 0.069 \\
\hline Hatchling survival & 1,71 & $1.157 \pm 0.848$ & 1.9 & 0.177 & $-0.655 \pm 0.770$ & 0.7 & 0.398 & - & - & - \\
\hline
\end{tabular}


Table 2.

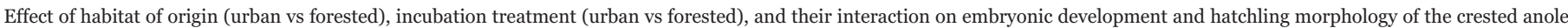

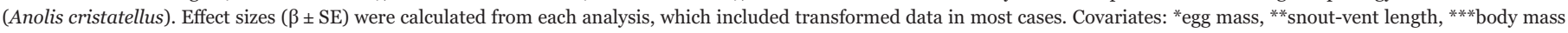
just prior to racing.

\begin{tabular}{|c|c|c|c|c|c|c|c|c|c|c|}
\hline \multirow[t]{2}{*}{ Dependent variable } & & \multicolumn{3}{|l|}{ Habitat } & \multicolumn{3}{|l|}{ Treatment } & \multicolumn{3}{|l|}{ Interaction } \\
\hline & df & $\beta \pm \mathrm{SE}$ & $\mathrm{F}$ & $\mathrm{p}$ & $\beta \pm \mathrm{SE}$ & $\mathrm{F}$ & $\mathrm{p}$ & $\beta \pm \mathrm{SE}$ & $\mathrm{F}$ & $\mathrm{p}$ \\
\hline Incubation Period & 1,88 & $-0.061 \pm 0.051$ & 2.7 & 0.105 & $0.182 \pm 0.015$ & 152.9 & $<0.001$ & $-0.039 \pm 0.026$ & 2.2 & 0.139 \\
\hline Egg survival & 1171 & $-0.055 \pm 0.538$ & 0.6 & 0.499 & $-0.760 \pm 0.396$ & 1.3 & 0.254 & $0.812 \pm 0.618$ & 1.7 & 0.190 \\
\hline Snout-vent length $(\mathrm{mm})^{*}$ & 1,85 & $-0.016 \pm 0.014$ & 0.7 & 0.399 & $0.017 \pm 0.011$ & 6.3 & 0.015 & $0.013 \pm 0.019$ & 0.5 & 0.502 \\
\hline Mass $(g)^{*}$ & 1,85 & $-0.053 \pm 0.030$ & 3.7 & 0.057 & $-0.036 \pm 0.020$ & 5.6 & 0.020 & $0.006 \pm 0.034$ & 0.0 & 0.853 \\
\hline Condition, Mass (g) ${ }^{* *}$ & 1,85 & $-0.029 \pm 0.034$ & 0.2 & 0.643 & $-0.001 \pm 0.021$ & 0.8 & 0.379 & $0.031 \pm 0.036$ & 0.7 & 0.391 \\
\hline Tail Length (mm) & 1,85 & $0.018 \pm 0.025$ & 0.8 & 0.364 & $-0.001 \pm 0.017$ & 0.0 & 0.995 & $0.002 \pm 0.029$ & 0.0 & 0.954 \\
\hline \multicolumn{11}{|l|}{ Running performance } \\
\hline Over $25 \mathrm{~cm}^{* * *}$ & 1,62 & $-0.214 \pm 0.238$ & 0.4 & 0.517 & $-0.284 \pm 0.196$ & 1.2 & 0.283 & $0.210 \pm 0.328$ & 0.4 & 0.524 \\
\hline Over $1 \mathrm{~m}^{* * *}$ & 1,56 & $-0.175 \pm 0.180$ & 0.8 & 0.365 & $-0.132 \pm 0.146$ & 0.3 & 0.572 & $0.124 \pm 0.244$ & 0.3 & 0.612 \\
\hline Number of stops & 1,63 & $-1.874 \pm 1.356$ & 2.3 & 0.136 & $-1.094 \pm 1.143$ & 0.5 & 0.490 & $0.865 \pm 1.905$ & 0.2 & 0.651 \\
\hline locomotor behavior & 1,63 & $-0.083 \pm 0.827$ & 0.1 & 0.803 & $-0.231 \pm 0.699$ & 0.3 & 0.618 & $-0.147 \pm 1.214$ & 0.1 & 0.904 \\
\hline Growth & 1,63 & $-0.001 \pm 0.001$ & 2.1 & 0.153 & $-0.001 \pm 0.0008$ & 3.5 & 0.067 & $-0.001 \pm 0.001$ & 1.8 & 0.185 \\
\hline Hatchling survival & 1,87 & $0.125 \pm 0.638$ & 0.3 & 0.587 & $0.170 \pm 0.539$ & 0.4 & 0.525 & $0.280 \pm 0.972$ & 0.1 & 0.774 \\
\hline
\end{tabular}

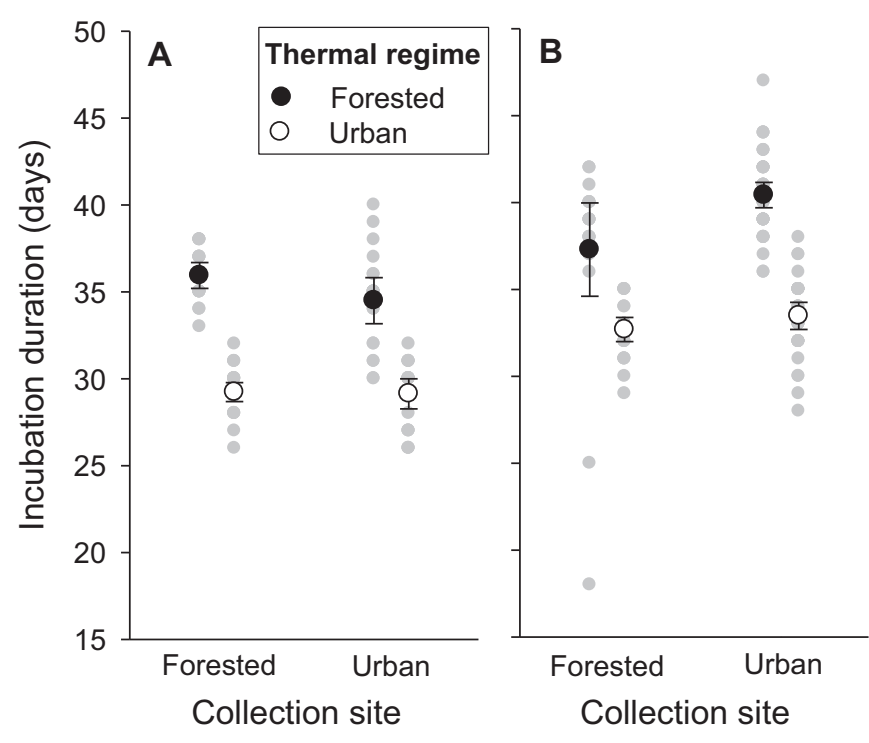

Fig. 2. Mean incubation duration and $95 \%$ confidence intervals for eggs from urban and forested locations that were incubated under temperatures that mimic nest sites at each site in Miami for (A) Anolis sagrei and (B) A. cristatellus. The gray points represent raw data.

performance ( $A$. sagrei, mean: 8.0 days old, range 6-16 days; $A$. cristatellus, mean: 7.5 days old, range 4-10 days) for both $A$. sagrei (87\%) and A. cristatellus (78\%). Variation in hatchling survival was not explained by the treatments or their interactions.

\section{Discussion}

Because reptile embryos are sensitive to temperature, the elevated thermal conditions in cities due to the urban heat island effect could influence embryonic development. To address whether embryos are physiologically adapted to urban nest temperatures, we quantified the effects of incubation temperatures on two non-native species of Anolis lizards that have established populations in both urban and forested areas in the Miami metropolitan area of south Florida. Our results demonstrate that the relatively warm temperatures of urban sites accelerate embryonic development (as seen in all reptiles; Deeming, 2004), but have no major consequences on morphology or locomotor performance of hatchlings. These results provide no evidence that embryos of $A$. sagrei or $A$. cristatellus from urban and forested sites are locally adapted to their respective thermal environments. Instead, the lack of any major effect of incubation temperature suggests that both these species are physiologically robust with respect to variation in nest temperatures.

Although we show no evidence of adaptation of embryos to the thermal environment of the nest, some results suggest that fitness may be enhanced in urban environments. First, the increased developmental rate of embryos under urban conditions suggests that eggs in urban sites have less time exposed to potentially adverse conditions or to predators compared to eggs in forested sites (Shine, 2002). Moreover, the reduced incubation length results in earlier hatching, which has been shown to positively affect survival in other lizard species (Olsson and Shine, 1997; Warner and Shine, 2007; Wapstra et al., 2010). These benefits are consistent with another study of an Anolis lizard that shows egg survival is higher and incubation length reduced in humanmodified habitat (pastures) compared to undisturbed forested habitat (Schlaepfer, 2003). In addition, although fecundity did not differ between urban and forested sites when females were housed in a common laboratory environment, previous work on A. cristatellus suggests that open/warmer environments could increase reproductive output by allowing females to remain reproductively active throughout the year (Otero et al., 2015). Indeed, the relatively warm conditions in cities may accelerate reproductive cycles in ways that increase egg output over the season; similar patterns have been shown in other organisms (Pörtner et al., 2001; Gotthard et al., 2007; but see Meineke et al. (2013)). These overall positive effects on developmental rate and fecundity in relatively warm environments may contribute to the success of $A$. sagrei and $A$. cristatellus in urban areas.

The lack of evidence for adaptation to site-specific incubation environments in terms of embryonic development and hatchling performance may be explained by multiple factors. First, the two forested sites in this study are only $1.2-1.6 \mathrm{~km}$ from the urban collection site, and gene flow between sites could prevent local adaptation (Slatkin, 1987; Lenormand, 2002). Second, even if gene flow was absent, maternal nest site choice could reduce differences in nest thermal environments (Doody et al., 2006; Refsnider and Janzen, 2012), thereby hindering thermal adaptation by embryos. At present, nest conditions of anoles are poorly understood, except that females use a variety of nest sites (e.g., under leaf litter, under logs, in tree holes; Rand, 1967; Andrews, 1988) and tend to prefer relatively moist conditions for nesting (Socci et al., 2005; Reedy et al., 2013). Although the temperatures of potential nest sites used in this study may not reflect the exact conditions that females choose, we still selected a variety of microhabitats that likely encompassed the range of temperatures that are selected by females. Notably, the forested site showed relatively low thermal variation (Fig. 1), which is indicative of the 
uniform and highly shaded habitat at this site. Overall, we are confident that the thermal regimes used in this experiment reflect ecologicallyrelevant conditions that eggs are likely to experience at each site. Even so, temperatures may not be extreme enough between urban and forested sites (possibly due to their close proximity) to elicit sitespecific responses despite large differences in canopy cover and ambient temperatures at these sites (Kolbe et al., 2016).

A third explanation for the lack of evidence for adaptation to sitespecific thermal environments may involve the timing and patterns of colonization to Miami, which could influence the degree to which populations are adapted to current environments. For example, both species have been present in Miami for relatively short periods ( $\sim 60$ years for A. sagrei and $\sim 40$ years for A. cristatellus), and it is unclear whether thermal adaptation of embryos is likely over these time periods. However, anoles can exhibit morphological adaptation to novel environments within this timeframe (Losos et al., 1997; Kolbe et al., 2012). Indeed, A. cristatellus exhibits morphological adaptations that confer better performance on artificial substrates in urban areas (Kolbe et al., 2015; Winchell et al., 2016). However, multiple introductions and admixture over time (Kolbe et al., 2004) in urban and forested sites could reduce the likelihood of any population-specific responses to urban or forested incubation temperatures. Importantly, the source populations for both these species were likely from urban environments within their native ranges (Kolbe et al., 2004, 2007), suggesting that adaptation to urban conditions may have preceded biological invasion. If adaptation prior to invasion occurred, then population-specific responses to urban incubation temperatures would not be expected in their non-native ranges. Studies of survival in urban and natural post-hatching environments will provide further insights into the potential for adaptation to urban incubation environments.

Paternal effects may also contribute to phenotypic variation in a way that reduces the effects of the incubation environment. For example, males in our breeding colony were rotated among females (but populations were never mixed), which could result in males siring offspring from more than one female. Our breeding design could potentially increase genetic relatedness (and reduce independence) among offspring, and therefore decrease variation in how embryos respond to different thermal environments. This possibility could have been eliminated if each female was paired with a different male over the duration of the experiment. Despite this possibility, however, it is unknown whether paternity was truly biased, as females can store viable sperm for several months (even from mating events in the field prior collection for to the experiment) (Calsbeek et al., 2007). Additionally, although our design may have influenced the order of mating events with males, past work on $A$. sagrei suggests that mating order has little impact on siring success (Calsbeek et al., 2007), and that paternal phenotype has little to no impact on offspring phenotypes (Warner et al., 2013; but see Cox et al. (2011)). Thus, paternal factors likely contributed little to variation in our dataset.

Overall, our study provides no evidence for adaptation to urban thermal environments by embryos of two non-native anole species in Miami, but suggests that urban conditions are conducive for successful establishment. Indeed, accelerated developmental rate and possible increased fecundity in urban environments suggest that these species may have already been well suited for urban thermal environments. Moreover, the lack of any site-specific phenotypic response to incubation temperature suggests that these species are robust to environmental changes. These factors likely contributed the success of $A$. sagrei and $A$. cristatellus in non-native ranges and urban environments. Studies that further explore the effects of novel early-life environments on fitness-relevant phenotypes will be critical in understanding establishment of populations in non-native ranges and in human-modified environments.

\section{Acknowledgements}

Thanks to James Stroud and Zachary Chejanovski for collecting and shipping lizards for this experiment. Thanks to C. Cates, T. Mitchell, P. Pearson, and D. Williams for assistance with lizard care and egg incubation. We thank Patrick Griffith for permission to collect lizards on plants at Montgomery Botanical Center. Miami-Dade Parks Natural Areas Management granted permission for us to work in Matheson Hammock Park (Permit \# 205U). This project was approved by the University of Alabama at Birmingham Institutional Animal Care and Use Committee (Protocol \# 130909976). ST received support from Gonzaga University's Off-Campus Research Experience Program, funded by a grant from the Howard Hughes Medical Institute. This project was support by the National Science Foundation (Grant \# DEB1354897 to JJK and DAW).

\section{Appendix A. Supporting information}

Supplementary data associated with this article can be found in the online version at doi:10.1016/j.jtherbio.2017.02.021.

\section{References}

Andrews, R.M., 1988. Demographic correlates of variable egg survival for a tropical lizard. Oecologia 76, 376-382.

Angilletta, M.J., 2009. Thermal Adaptation: A Theoretical and Empirical Synthesis. Oxford University Press, Oxford.

Angilletta, M.J., Niewiarowski, P.H., Navas, C.A., 2002. The evolution of thermal physiology in ectotherms. J. Therm. Biol. 27, 249-268.

Angilletta, M.J., Oufiero, C., Sears, M., 2004. Thermal adaptation of maternal and embryonic phenotypes in a geographically widespread ectotherm. Int. Conf. Ser. 1275, 258-266.

Brazel, A., Selover, A., Vose, R., Heisler, G., 2000. The tale of two climates - Baltimore and Pheonix urban LTER sites. Clim. Res. 15, 123-135.

Calsbeek, R., Bonneaud, C., Prabhu, S., Manoukis, N., Smith, T.B., 2007. Multiple paternity and sperm storage lead to increased genetic diversity in Anolis lizards. Evol. Econ. Res. 9, 495-503.

Conover, D.O., Schultz, E.T., 1995. Phenotypic similarity and the evolutionary significance of countergradient variation. Trends Ecol. Evol. 10, 248-252.

Cox, R.M., Duryea, M.C., Najarro, M., Calsbeek, R., 2011. Paternal condition drives sexratio bias in a lizard that lacks parental care. Evolution 65, 220-230.

Deeming, D., 2004. Reptilian Incubation: Environment, Evolution, and Behaviour. NottinghamUniversity Press, Nottingham.

Doody, J., Guarino, E., Georges, A., Corey, B., Murray, G., Ewert, M., 2006. Nest site choice compensates for climate effects on sex ratios in a lizard with environmental sex determination. Evol. Ecol. 20, 307-330.

Du, W., Warner, D., Langkilde, T., Robbins, T., Shine, R., 2010. The physiological basis of geographic variation in rates of embryonic development within a widespread lizard species. Am. Nat. 176, 522-528.

Ewert, M., Lang, J., Nelson, C., 2005. Geographic variation in the pattern of temperaturedependent sex determination in the American snapping turtle (Chelydra serpentina). J. Zool. 265, 81-95.

Forman, R.T.T., 2014. Urban Ecology. Cambridge University Press, Cambridge.

Frankie, G.W., Ehler, L.E., 1978. Ecology of insects in urban environments. Ann. Rev. Entomol. 23, 367-387.

Gotthard, K., Berger, D., Walters, R., 2007. What keeps insects small? Time limitation during oviposition reduces the fecundity benefit of female size in a butterfly. Am. Nat. 169, 768-779.

Hamer, A.J., Parris, K.M., 2011. Local and landscape determinants of amphibian communities in urban ponds. Ecol. Appl. 21, 378-390.

Huey, R., Hertz, P., Sinervo, B., 2003. Behavioral drive versus behavioral inertia in evolution: a null model approach. Am. Nat. 161, 357-366.

Kaiser, A., Merckx, T., Van Dyck, H., 2016. The urban heat island and its spatial scale dependent impact on survival and development in butterflies of different thermal sensitivity. Ecol. Evol. 6, 4129-4140.

Kolbe, J.J., Glor, R.E., Roodriguez Schettino, L., Chamizo Lara, A., Larson, A., Losos, J.B., 2004. Genetic variation increases during biological invasion by a Cuban lizard. Nature 431, 177-181.

Kolbe, J.J., Glor, R.E., Rodriguez-Schettino, L., Chamizo-Lara, A., Larson, A., Losos, J.B., 2007. Multiple sources, admixture, and genetic variation in introduced Anolis lizard populations. Conserv. Biol. 21, 1612-1625.

Kolbe, J.J., VanMiddlesworth, P.S., Losin, N., Dappen, N., Losos, J.B., 2012. Climatic niche shift predicts thermal trait response in one but not both introductions of the Puerto Rican lizard Anolis cristatellus to Miami, Florida, USA. Ecol. Evol. 2, 1503-1516.

Kolbe, J., Ehrenberger, J., Moniz, H., Angilletta, M.J., 2014. Physiological variation among invasive populations of the brown anole (Anolis sagrei). Physiol. Biochem. Zool. 87, 92-104.

Kolbe, J., Battles, A., Avilés-Rodríguez, K., 2015. City slickers: poor performance does 
not deter Anolis lizards from using artificial substrates in human-modified habitats. Funct. Ecol.. http://dx.doi.org/10.1111/1365-2435.12607.

Kolbe, J.J., VanMiddlesworth, P., Battles, A.C., Stroud, J.T., Buffum, B., Forman, R.T.T., Losos, J.B., 2016. Determinants of spread in an urban landscape by an introduced lizard. Land. Ecol. doi:http://dx.doi.org/10.1007/s10980-016-0362-1.

Lenormand, T., 2002. Gene flow and the limits to natural selection. Trends Ecol. Evol. 17, 183-189.

Losos, J.B., Warheit, K.I., Schoener, T.W., 1997. Adaptive differentiation following experimental island colonization in Anolis lizards. Nature 387, 70-73.

Losos, J.B., Schoener, T.W., Spiller, D.A., 2004. Predator-induced behaviour shifts and natural selection in field-experimental lizard populations. Nature 432, 505-508.

Meineke, E.K., Dunn, R.R., Sexton, J.O., Frank, S.D., 2013. Urban warming drives insect pest abundance on street trees. PLoS One. http://dx.doi.org/10.1371/ journal.pone.0059687.

Menke, S., Guénard, B., Sexton, J., Weiser, M., Dunn, R., Silverman, J., 2011. Urban areas may serve as habitat and corridors for dry-adapted, heat tolerant species; an example from ants. Urban Ecosyst. 14, 135-163.

Niewiarowski, P., Angilletta, M.J., 2008. Countergradient variation in embryonic growth and development: do embryonic and juvenile performances trade off? Funct. Ecol. 22, 895-901.

Olsson, M., Shine, R., 1997. The seasonal timing of oviposition in sand lizards (Lacerta agilis): why early clutches are better. J. Evol. Biol. 10, 369-381.

Otero, L.M., Huey, R.B., Gorman, G.C., 2015. A few meters matter: local habitats drive reproductive cycles in a tropical lizard. Am. Nat. 186, E72-E80.

Oufiero, C.E., Angilletta, M.J., 2006. Convergent evolution of embryonic growth and development in the eastern fence lizard (Sceloporus undulatus). Evolution 60, 1066-1075.

Pörtner, H.O., Berdal, B., Blust, R., Brix, O., Colosimo, A., De Wachter, B., Giuliani, A., Johansen, T., Sischer, T., Knust, R., Lannig, G., Naevdal, G., Nedenes, A., Nyhammer, B., Sartoris, F.J., Serendero, I., Sirabella, P., Thorkildsen, S., Zakhartsev, M., 2001. Climate induced temperature effects on performance, fecundity and recruitment in marine fish: developing a hypothesis for cause and effect relationships in Atlantic cod (Gadus morhua) and common eelpout (Zoarces viviparus). Cont. Shelf Res. 21, 1975-1997.

Rand, A.S., 1967. Communal egg laying in anoline lizards. Herpetologica 23, 227-230. Reedy, A.M., Zaragoza, D., Warner, D.A., 2013. Maternally-chosen nest sites positively affect multiple components of offspring fitness in lizards. Behav. Ecol. 24, 39-46. Refsnider, J.M., Janzen, F.J., 2012. Behavioral plasticity may compensate for climate change in a long-lived reptile with temperature-dependent sex determination. Biol. Conserv. 152, 90-95.

Rizwan, A.M., Dennis, Y.C.L., Liu, C., 2008. A review of the generation, determination and mitigation of urban heat island. J. Environ. Sci. 20, 120-128.

Schlaepfer, M.A., 2003. Successful lizard eggs in a human-disturbed habitat. Oecologia 137, 304-311.

Shochat, E., Warren, P.S., Faeth, S.H., McIntyre, N.E., Hope, D., 2006. From patterns to emerging processes in mechanistic urban ecology. Trends Ecol. Evol. 21, 186-191.

Shine, R., 2002. Eggs in autumn: responses to declining incubation temperatures by eggs of montane lizards. Biol. J. Linn. Soc. 76, 71-77.

Slatkin, M., 1987. Gene flow and the geographic structure of natural populations. Science 236, 787-792.

Socci, A.M., Schlaepfer, M.A., Gavin, T.A., 2005. The importance of soil moisture and leaf cover in a female lizard's (Norops polylepis) evaluation of potential nest sites. Herpetologica 61, 233-240.

Stuart, Y., Campbell, T., Hohenlohe, P., Reynolds, R., Revell, L., Losos, J.B., 2014. Rapid evolution of a native species following invasion by a congener. Science 346, 463-466.

Telemeco, R.S., Gangloff, E.J., Cordero, G.A., Mitchell, T.S., Bodensteiner, B.L., Holden, K.G., Mitchell, S.M., Polich, R.L., Janzen, F.J., 2016. Reptile embryos lack the opportunity to thermoregulate by moving within the egg. Am. Nat. 188, E13-E27.

Tereshchenko, I.E., Filonov, A.E., 2001. Air temperature fluctuations in Guadalajara, Mexico, from 1926 to 1994 in relation to urban growth. Int. J. Climatol. 21, 483-494.

Wapstra, E., Uller, T., While, G.M., Olsson, M., Shine, R., 2010. Giving offspring a head start in life: field and experimental evidence for selection on maternal basking behaviour in lizards. J. Evol. Biol. 23, 651-657.

Warner, D.A., Shine, R., 2007. Fitness of juvenile lizards depends on seasonal timing of hatching, not offspring body size. Oecologia 154, 65-73.

Warner, D.A., Kelly, C.D., Lovern, M.B., 2013. Reproductive experience affects mating behaviour, but does not impact parental allocation in a lizard. Behav. Ecol. Sociobiol. 67, 973-983.

Winchell, K., Reynolds, R.G., Prado-Irwin, S., Puente-Rolón, A., Revell, L., 2016. Phenotypic shifts in urban areas in the tropical lizard Anolis cristatellus. Evolution 70, 1009-1022. 